\title{
Sip size of orangeade: effects on intake and sensory-specific satiation
}

\author{
Pascalle L. G. Weijzen ${ }^{1,2}$, Paul A. M. Smeets ${ }^{1,3}$ and Cees de Graaf ${ }^{1}$ \\ ${ }^{1}$ Division of Human Nutrition, Wageningen University, PO Box 8129, 6700 EV Wageningen, The Netherlands \\ ${ }^{2}$ FrieslandCampina Corporate Research, PO Box 87, 7400 AB Deventer, The Netherlands \\ ${ }^{3}$ Image Sciences Institute, University Medical Centre Utrecht, Utrecht, The Netherlands
}

(Received 1 December 2008 - Revised 11 February 2009 - Accepted 10 March 2009 - First published online 15 April 2009)

Sensory-specific satiation (SSS) drives food selection and contributes to meal termination. We hypothesised that smaller sips would increase SSS due to increased oro-sensory exposure, irrespective of energy content. The objective was to determine the effects of sip size and energy content on ad libitum intake of orangeade and subjective SSS for orangeade. Orangeade intake and ratings of wanting and liking were measured before and after ad libitum orangeade consumption in a $2 \times 2$ cross-over design $(n 53)$. Conditions differed in energy content (no-energy $v$. regular-energy orangeade) and in sip size (large, $20 \mathrm{~g} / \mathrm{sip} v$. small, $5 \mathrm{~g} / \mathrm{sip}$ ). The mean intake of both orangeades was lower when consumed with small sips than when consumed with large sips (regular-energy, 352 v. $493 \mathrm{~g}$; no-energy, $338 v .405 \mathrm{~g}$; both $P<0 \cdot 001$ ). When consumed with large sips, the mean intake of no-energy orangeade was lower than that of regular-energy orangeade $(P=0.02)$. When consumed with small sips, subjective SSS (based on the desire to drink) was higher for no-energy orangeade than for regular-energy orangeade $(P=0 \cdot 01)$, while mean intake was comparable. We concluded that smaller sip size, i.e. increased oro-sensory exposure per unit of consumption, can lower intake of sweet drinks. Only with low oro-sensory exposure (large sip size) was intake higher for an energy-containing sweet drink than for a no-energy sweet drink. This suggests that intake of sweet drinks is stimulated by (metabolic) reward value and inhibited by sensory satiation. This underpins the importance of SSS for meal termination.

Sensory-specific satiation: Intake: Sip size: Energy content

The regulation of food choice is governed by internal factors, such as a specific subconscious desire for the food, an overt liking, and/or habit (for a review, see $\mathrm{Mela}^{(1)}$ ), and by environmental factors, such as serving size and social context (for a review, see Wansink ${ }^{(2)}$ ). Whatever has prompted the choice, as soon as food enters the mouth, the taste and smell of food generate the first triggers of satiation, through their action on sensory receptors ${ }^{(3)}$. Sensory-specific satiation (SSS) results from continued sensory stimulation and has been defined as the decline in wanting or liking of a food relative to uneaten foods ${ }^{(4)}$. SSS has been shown to contribute to food intake control (for a review, see Sørensen et al. ${ }^{(5)}$ ).

Factors thought to influence food intake and the degree of SSS for a food are its intensity ${ }^{(6)}$ and the duration ${ }^{(7)}$ of oro-sensory exposure. The shorter oro-sensory exposure to liquids could explain the low satiating power of liquid energy as compared with energy from solid foods ${ }^{(8,9)}$, and the weak compensatory response after consumption of liquid energy ${ }^{(10-12)}$. Also the bite or sip size by which a given food is consumed influences the duration of oro-sensory exposure. When consumed with smaller bites or sips, a smaller amount per chew ${ }^{(13,14)}$ or per swallow ${ }^{(15)}$ is ingested, which results in a longer oro-sensory exposure per consumption.

Another factor that potentially influences SSS is the energy content of food. Although some studies showed that the degree of SSS increased when the energy content or amount of solid foods ingested increased ${ }^{(16,17)}$, studies that dissociated the sensory properties of fat or sugar from the energy they provided, while matching the food stimuli for taste and texture, found that the degree of SSS did not depend on energy content ${ }^{(18-21)}$.

The present study tested whether (1) consumption of orangeade with smaller sips leads to a lower ad libitum intake and a higher degree of subjective SSS, and whether (2) orangeade intake and the degree of subjective SSS are affected by the energy content of similarly sweet orangeades.

\section{Subjects and methods}

\section{Subjects}

Subjects were fifty-three healthy adults (twenty-one male, thirty-two female), aged 18-29 (mean 21.9) years. The mean BMI was 21.5 (SD 1.7) $\mathrm{kg} / \mathrm{m}^{2}$. Subjects were recruited from a consumer database of the division of Human Nutrition of Wageningen University. A pre-screening questionnaire was performed by eligible subjects to confirm that they had normal weight (BMI $18.5-25.0 \mathrm{~kg} / \mathrm{m}^{2}$ ), their weight was stable (no weight change of more than $5 \mathrm{~kg}$ during the previous 6 months), did not smoke, did not have a gastrointestinal

Abbreviations: GLM, general linear model; LSD, least significant difference; SSS, sensory-specific satiation.

* Corresponding author: Dr Pascalle Weijzen, fax +31 570695 918, email Pascalle.weijzen@frieslandcampina.com 
illness, an illness of the thyroid gland or diabetes, did not have a food allergy, were not pregnant or lactating, did not use medication likely to affect taste perception or appetite, were not highly dietary restrained according to the norm tables of the Dutch Eating Behaviour Questionnaire (DEBQ) $)^{(22)}$, and were consumers of soft drinks (at least $1 \mathrm{~d} / \mathrm{month}$ ). Subjects were naive to the aim of the research and blind concerning the treatments. Subjects provided informed consent and were reimbursed for participation. The protocol of the study was approved by the Medical Ethical Committee of Wageningen University.

\section{Study design}

The study design was a balanced $2 \times 2$ (sip size $\times$ energy) single-blind cross-over design. For each subject the time between two sessions was at least $48 \mathrm{~h}$. Each session, one of two types of orangeade (regular-energy or no-energy orangeade) was consumed in one of two sip size conditions (large or small sips). The regular-energy orangeade was sweetened with sucrose, while the no-energy orangeade was sweetened with synthetic sweeteners. The orangeades were consumed through a silicon tube (length $1.5 \mathrm{~m}$; diameter $5 \mathrm{~mm}$ ) that was connected to a peristaltic pump (HR flow inducer, MHRE 200; Watson-Marlow Ltd, Falmouth, Cornwall, UK), whereby the sip size, the delivery rate, and the interval between the sips were controlled. In the large sip condition, large sips $(20 \mathrm{~g} / \mathrm{sip})$ were delivered at a high rate $(600 \mathrm{~g} / \mathrm{min})$ for $2 \mathrm{~s}$, and with large intervals between sips (6s). In the small sip condition, small sips $(5 \mathrm{~g} / \mathrm{sip})$ were delivered at a low rate $(300 \mathrm{~g} / \mathrm{min})$ for $1 \mathrm{~s}$, and with small intervals between sips ( $1 \mathrm{~s})$. In both conditions the ingestion rate was $150 \mathrm{~g} / \mathrm{min}$. Subjects were instructed to swallow immediately after each sip. Therefore, in the small sip condition the duration of oro-sensory exposure per consumption, i.e. the interval between the food entering the mouth and swallowing, was higher than in the large sip condition $( \pm 30 \mathrm{~s} / \mathrm{min}$ in the small sip condition $v .15 \mathrm{~s} / \mathrm{min}$ in the large sip condition). In a pilot study performed before the outset of the study in a small group of subjects not participating in the study $(n 10)$ it was ensured that subjects perceived a clear difference in the duration of oro-sensory exposure between the two sip size conditions, while it was reasonably pleasant to consume the orangeades in each of the conditions.

The study design was similar to that of previous SSS studies (for example, Rolls et al. ${ }^{(4)}$ ). During each session, subjects first rated their appetite. Then, they tasted small samples $( \pm 15 \mathrm{~g})$ of three stimuli, one of the two orangeades, and two reference stimuli, semi-skimmed milk and tomato juice. Milk and tomato juice were chosen as reference stimuli, because they clearly differed in taste from the orangeades.
Subjects rated the stimuli on four measures: two measures of wanting, i.e. desire to eat and prospective consumption, one measure of liking, i.e. pleasantness, and sweetness intensity. Subsequently, they consumed an ad libitum amount of the rated orangeade in one of the two sip size conditions. They received no visual cues on the amount ingested. Next, they gave appetite ratings again. Finally, they once more tasted small samples of the same three stimuli and rated them on the same four measures (Fig. 1).

\section{Stimuli}

The orangeades were prepared from orange-flavoured syrups. These were diluted with water $(1: 5 \mathrm{~g})$. The syrups were specifically prepared for the study by United Soft Drinks (Utrecht, The Netherlands), and were matched for sweetness, viscosity and appearance. One of the syrups was sweetened with sucrose (per $100 \mathrm{~g}$ orangeade: $10.4 \mathrm{~g}$ sucrose, $177 \mathrm{~kJ}$ ); the other syrup was sweetened with a combination of synthetic sweeteners (per $100 \mathrm{~g}$ orangeade: $0.011 \mathrm{~g}$ aspartame, $0.011 \mathrm{~g}$ acesulfame $\mathrm{K}, 0.0058 \mathrm{~g}$ sodium cyclamate, $0.0015 \mathrm{~g}$ sodium saccharin, $\pm 0 \mathrm{~kJ})$.

The two reference stimuli of the rating set were chosen to clearly differ in taste from the orangeades. These stimuli were tomato juice (Appelsientje Zontomaat; Riedel ${ }^{\circledR}$, Ede, The Netherlands) and ultra-heat-treated sterilised semiskimmed milk (Langlekker; Friesche Vlag ${ }^{\circledR}$, Nijkerk, The Netherlands). All stimuli were consumed at room temperature.

\section{Procedure}

On the test days subjects came to the sensory laboratory. They were instructed to eat a normal breakfast and lunch, and not to eat anything for $2 \mathrm{~h}$ before a test session (except for water, and tea or coffee without added milk or sugar), to make sure that feelings of hunger were comparable among test days. This was confirmed by baseline measures of appetite on each test day.

The study was performed in tasting booths. As soon as the subjects arrived at a test session they received oral and written instructions concerning the procedure of the test session. Next, they completed an appetite questionnaire of five items (hunger, thirst, fullness, desire to eat, desired amount to eat). The items were rated on nine-point scales, labelled 'not at all' (1) and 'extremely' (9). In order to determine their habitual sip size, subjects were asked to drink three sips of water. Subsequently, subjects were presented with a tray containing small samples of the three stimuli. They were asked to taste and rate the stimuli from left to right. The presentation order of the samples on the tray was randomised across subjects and across sessions. Subjects evaluated each
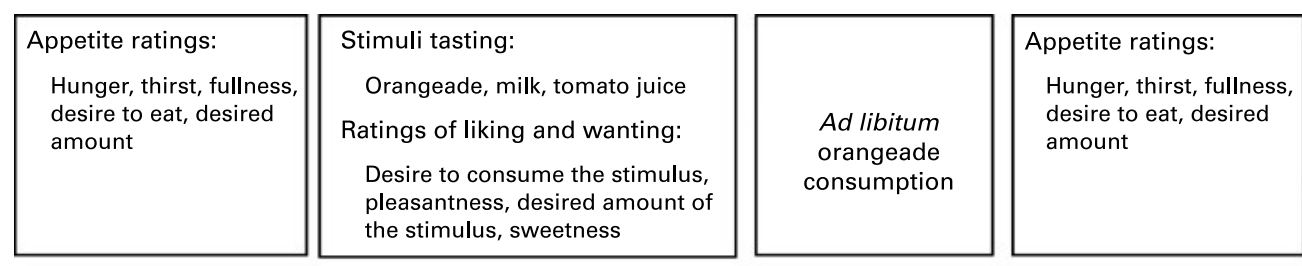

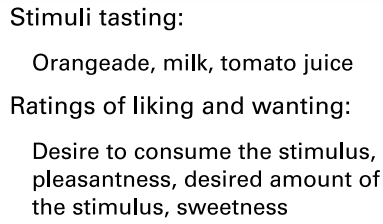

Fig. 1. Schematic overview of the design of each of the four experimental sessions for a subject. The ad libitum consumed orangeade was either no-energy or regular-energy orangeade, and was either consumed with small $(5 \mathrm{~g})$ or with large $(20 \mathrm{~g})$ sips. 
stimulus with respect to their desire to consume the stimulus, pleasantness, prospective consumption, and perceived sweetness. These items were also rated on nine-point scales, labelled 'not at all' (1) and 'extremely' (9). Subjects were instructed to rinse their mouth with water between tasting the different stimuli.

Next, subjects were instructed to take the tip of the tube in their mouth, and to start the pump. This started the delivery of orangeade. They were instructed to consume the orangeade until they felt they had had enough. They were allowed to stop and re-start the pump whenever they wanted. To avoid subjects from terminating consumption because of time reasons, the time for consumption was held constant at $10 \mathrm{~min}$ for all participants. After $10 \mathrm{~min}$, subjects repeated completion of the appetite questionnaire, and subsequently the same tasting and rating procedure as before consumption of the orangeade.

\section{Statistical analysis}

Statistical analyses were performed using SPSS (version 12.0.1; SPSS, Inc., Chicago, IL, USA). Effects were considered statistically significant at a value of $P<0 \cdot 05$.

For data analysis subjects' habitual soft drink consumption was categorised in three categories: (1) regular-energy soft drink consumers, indicated to consume regular-energy soft drinks more often than no-energy soft drinks (37\%); (2) both regular-energy and no-energy soft drink consumers, indicated to consume regular-energy and no-energy soft drinks equally often $(28 \%)$; (3) no-energy soft drink consumers, indicated to consume no-energy soft drinks more often than regularenergy soft drinks $(35 \%)$. The average habitual sip size of each subject was categorised into two categories, (1) large $(\geq 23.3 \mathrm{~g})$ or $(2)$ small $(<23.3 \mathrm{~g})$, on the basis of a median split of the average sip size of water of all subjects.

Ad libitum intake was compared among the four experimental conditions by the repeated-measures procedure of the general linear model (GLM) with sip size and energy as within-subject factors, and sex, habitual sip size, and soft drink consumer type as between-subjects factors. The analysis was corrected for multiple comparisons by a Fisher's least significant difference (LSD) test.

Initial appetite ratings were compared among the four experimental conditions by the repeated-measures procedure of the GLM, for each of the five ratings. Changes in appetite ratings were compared among the four experimental conditions by the repeated-measures procedure of the GLM with sip size and energy as within-subject factors. The analysis was corrected for multiple comparisons by a Fisher's LSD test.

Initial ratings of wanting, liking, and perceived sweetness, averaged over the experimental conditions, were compared among each of the orangeades and each of the reference stimuli (milk and tomato juice) through the repeated-measures procedure of GLM. The analysis was corrected for multiple comparisons by a Fisher's LSD test.

To test whether the ratings of sweetness of the orangeades had changed after orangeade consumption for each of the four experimental conditions the pre-consumption ratings were compared with the post-consumption ratings by Student's $t$ tests for dependent samples.
Changes in ratings of wanting and liking from before to after orangeade consumption were compared between the consumed orangeades and each of the reference stimuli (milk and tomato juice), for each of the four experimental conditions (post-consumption - pre-consumption). This was done by the repeated-measures procedure of the GLM. The analysis was corrected for multiple comparisons by a Fisher's LSD test.

Subjective SSS was calculated by subtracting the changes in ratings of wanting (two measures) and liking (one measure) of orangeade from before to after orangeade consumption from the corresponding average changes in ratings of the reference stimuli (milk and tomato juice). Thus, for each of the four experimental conditions three measures of subjective SSS were calculated: (1) based on ratings of desire to drink, (2) based on pleasantness ratings, and (3) based on prospective consumption ratings. The more negative scores were, the higher subjective SSS was.

The degree of subjective SSS was compared for each of the three measures among the four experimental conditions by the repeated-measures procedure of the GLM with sip size and energy as within-subject factors, and sex, habitual sip size, and soft drink consumer type as between-subjects factors. The analysis was corrected for multiple comparisons by a Fisher's LSD test.

\section{Results}

The results were not statistically different between male and female subjects and between those who normally consumed beverages with small or large sips. Therefore, only the results of the uncorrected models (no between-subjects factors included) will be shown. The association between subjective SSS based on the desire to drink and orangeade type was different for regular-energy and no-energy soft drink consumers (i.e. orangeade type $\times$ normal soft drink consumer type interaction). For that outcome the results of the corrected model (corrected for normal soft drink consumer type, sex, and habitual sip size) will be shown.

\section{Ad libitum intake}

Figure 2 shows that when consumed with small sips, the mean intake of the orangeades was lower than when consumed with large sips (main effect sip size: $F(1,49) 40 \cdot 0 ; P<0 \cdot 001$ ). The effect of sip size was larger for the regular-energy orangeade than for the no-energy orangeade (regular-energy: mean intake $141 \mathrm{~g}(29 \%)$ lower in small sip condition; no-energy: mean intake $66 \mathrm{~g}(16 \%)$ lower in small sip condition (intake $X$ energy interaction: $F(1,49) 3 \cdot 7 ; P=0.06)$ ). This means that in the large sip condition mean intake was $88 \mathrm{~g}(18 \%)$ lower for the no-energy orangeade than for the energycontaining orangeade, while in the small sip condition intake was comparable between the two orangeade types.

\section{Initial ratings of wanting, liking and sweetness}

The initial ratings of wanting, liking and perceived sweetness did not differ between the two orangeade types, and were higher for the orangeades than for the milk and the tomato juice (Table 1). 


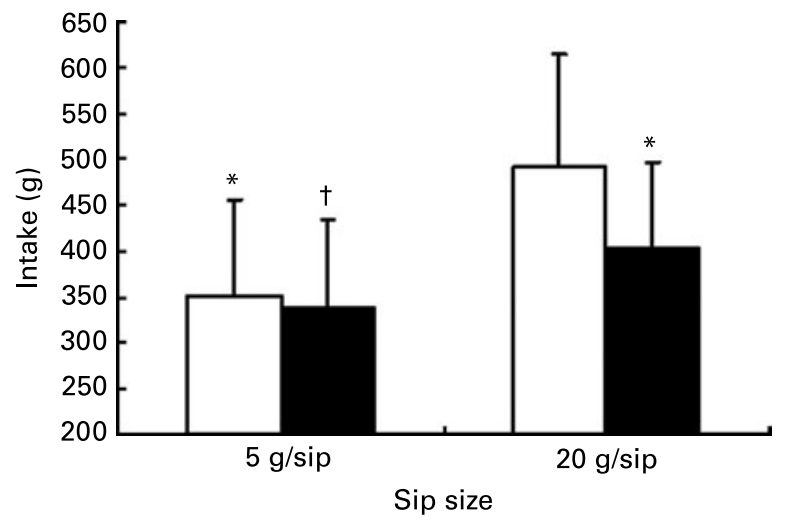

Fig. 2. Ad libitum intake (g) of the two types of orangeade ( $\square$, regular-energy; 口, no-energy) in the two sip size conditions. Values are means, with standard deviations represented by vertical bars. Fisher's least significant difference (LSD) multiple comparisons were made among the four experimental conditions. ${ }^{*}$ Mean value was significantly different from that of the regular-energy, $20 \mathrm{~g} / \mathrm{sip}$ condition $(P<0.05$; Fisher's LSD test). † Mean value was significantly different from that of the no-energy, $20 \mathrm{~g} / \mathrm{sip}$ condition $(P<0.05$; Fisher's LSD test).

\section{Subjective sensory-specific satiation}

Table 2 shows that the decline in subjective ratings of desire to consume and prospective consumption from before to after orangeade consumption was in each of the four conditions larger for the consumed orangeade than for the non-consumed reference stimuli. A similar pattern was observed for subjective ratings of pleasantness, except for in the small sip condition, in which the decline in pleasantness was comparable between the regular-energy orangeade and the non-consumed reference stimuli. Thus, sensory satiation was perceived for each of the orangeades in each of the sip size conditions, except for when based on ratings of pleasantness.

Subjective SSS (based on desire to drink, pleasantness, prospective consumption) did not significantly differ between the two sip size conditions. This applied for both types of orangeade (Fig. 3). In the small sip condition, but not in the large sip condition $(F(1,49) 4.8 ; P=0.03)$, subjective SSS based on the desire to drink was higher (i.e. more negative score) for the no-energy orangeade than for the regularenergy orangeade. A significant effect was found for the energy $\times$ soft drink consumer type interaction $(F(1,49) 4 \cdot 3$;
$P=0 \cdot 05)$, which means that this was specifically the case for the no-energy soft drink consumers, while for the regularenergy soft drink consumers perceived sensory satiation was similar for both types of orangeade. The other measures of subjective SSS were in both sip size conditions comparable between the two types of orangeade (Fig. 3).

\section{Initial ratings of appetite and changes in appetite after orangeade consumption}

All initial ratings of appetite (including thirst ratings) were comparable across the four experimental conditions. For declines in ratings of hunger and desired amount to consume from before to after ad libitum orangeade consumption, significant sip size $\times$ energy effects were found (hunger: $F(1,49) 5 \cdot 1, P=0 \cdot 03$; desired amount to consume: $F(1,49)$ $8 \cdot 4, P=0 \cdot 01)$. This means that for the energy-containing orangeade, but not for the no-energy orangeade, the declines in hunger $(P=0.03)$ and desired amount to consume $(P=0.02)$ were larger in the large sip condition than in the small sip condition. This is probably due to the larger difference in amount consumed between sip sizes for the energycontaining orangeade than for the no-energy orangeade. Moreover, in the large sip condition, ratings of desired amount to consume declined more for the regular-energy than for the no-energy orangeade $(P=0 \cdot 01)$. This is probably due to the larger amount consumed of the energy-containing orangeade compared with the no-energy orangeade in that condition (Table 3).

\section{Changes in ratings of sweetness after orangeade consumption}

The ratings of perceived sweetness of the orangeades did not change after consumption of the orangeades, except for the perceived sweetness of the no-energy orangeade in the $20 \mathrm{~g} / \mathrm{sip}$ condition, which increased after consumption (mean increase $0 \cdot 4 ; t(52) 3 \cdot 7 ; P=0 \cdot 001)$.

\section{Discussion}

In line with our expectations, we found that subjects consumed less of the orangeades when consumed with small sips. This was especially the case for the regular-energy

Table 1. Initial ratings of wanting and liking, and initial ratings of sweetness of the samples averaged over the experimental conditions*

(Mean values and standard deviations)

\begin{tabular}{|c|c|c|c|c|c|c|c|c|c|c|}
\hline & \multicolumn{2}{|c|}{$\begin{array}{c}\text { Regular- } \\
\text { energy } \\
\text { orangeade }\end{array}$} & \multicolumn{2}{|c|}{$\begin{array}{l}\text { No-energy } \\
\text { orangeade }\end{array}$} & \multicolumn{2}{|c|}{ Tomato juice } & \multicolumn{2}{|c|}{ Milk } & \multirow[b]{2}{*}{$\mathrm{F} \dagger$} & \multirow[b]{2}{*}{$P$} \\
\hline & Mean & SD & Mean & SD & Mean & SD & Mean & SD & & \\
\hline Initial desire & $6 \cdot 2^{b}$ & $1 \cdot 7$ & $6 \cdot 0^{\mathrm{b}}$ & 1.5 & $4 \cdot 0^{a}$ & 1.9 & $4 \cdot 1^{a}$ & 1.8 & 33.0 & $<0.001$ \\
\hline Initial pleasantness & $6 \cdot 4^{\mathrm{b}}$ & 1.4 & $6 \cdot 2^{\mathrm{b}}$ & $1 \cdot 2$ & $4 \cdot 3^{a}$ & $2 \cdot 0$ & $4 \cdot 8^{\mathrm{a}}$ & $2 \cdot 0$ & $16 \cdot 7$ & $<0.001$ \\
\hline Initial prospective consumption & $5 \cdot 4^{\mathrm{b}}$ & 1.4 & $5 \cdot 4^{\mathrm{b}}$ & 1.5 & $3 \cdot 4^{\mathrm{a}}$ & $1 \cdot 8$ & $3 \cdot 8^{a}$ & $1 \cdot 8$ & $28 \cdot 4$ & $<0.001$ \\
\hline Initial sweetness & $6 \cdot 8^{\mathrm{b}}$ & $1 \cdot 1$ & $6 \cdot 9^{b}$ & $1 \cdot 0$ & $3 \cdot 9^{\mathrm{a}}$ & $1 \cdot 6$ & $3 \cdot 5^{\mathrm{a}}$ & 1.4 & $141 \cdot 6$ & $<0.001$ \\
\hline
\end{tabular}

\footnotetext{
a,b Mean values within a row with unlike superscript letters were significantly different $(P<0.05)$.

${ }^{*}$ Ratings on a nine-point scale anchored from $1=$ not at all desire/pleasant $/$ much/sweet to $9=$ extremely desire/ pleasant $/ \mathrm{much} / \mathrm{sweet}$.

$\dagger F$ value of the ANOVA comparing the initial subjective ratings for each stimulus. All tests have $3,156 \mathrm{df}$.
} 
Table 2. Decline in ratings of wanting (desire and prospective consumption) and liking (pleasantness) of orangeade and the two reference samples from before to after (post-consumption - pre-consumption) ad libitum consumption of orangeade in each of the four different experimental conditions*

(Mean values and standard deviations)

\begin{tabular}{|c|c|c|c|c|c|c|c|c|}
\hline & \multicolumn{2}{|c|}{ Orangeade } & \multicolumn{2}{|c|}{ Tomato juice } & \multicolumn{2}{|c|}{ Milk } & \multirow[b]{2}{*}{$\mathrm{F \dagger}$} & \multirow[b]{2}{*}{$P$} \\
\hline & Mean & SD & Mean & SD & Mean & SD & & \\
\hline \multicolumn{9}{|l|}{ Desire } \\
\hline Regular-energy, $5 \mathrm{~g} / \mathrm{sip}$ & $-2.5^{b}$ & $-1 \cdot 8$ & $-1 \cdot 2^{a}$ & 1.4 & $-0.9^{a}$ & $1 \cdot 7$ & $13 \cdot 8$ & $<0.001$ \\
\hline Regular-energy, $20 \mathrm{~g} / \mathrm{sip}$ & $-3 \cdot 2^{b}$ & 1.6 & $-1 \cdot 1^{\mathrm{a}}$ & 1.7 & $-1 \cdot 2^{\mathrm{a}}$ & 1.6 & 28.5 & $<0.001$ \\
\hline No-energy, $5 \mathrm{~g} / \mathrm{sip}$ & $-3 \cdot 3^{b}$ & 1.3 & $-1.0^{\mathrm{a}}$ & 1.7 & $-0.8^{a}$ & $1 \cdot 7$ & $46 \cdot 3$ & $<0.001$ \\
\hline No-energy, $20 \mathrm{~g} / \mathrm{sip}$ & $-2 \cdot 8^{b}$ & $1 \cdot 7$ & $-0.9^{\mathrm{a}}$ & 1.4 & $-1 \cdot 1^{\mathrm{a}}$ & $1 \cdot 7$ & $23 \cdot 0$ & $<0.001$ \\
\hline \multicolumn{9}{|l|}{ Prospective consumption } \\
\hline Regular-energy, $5 \mathrm{~g} / \mathrm{sip}$ & $-2 \cdot 8^{b}$ & 1.8 & -1.2 & $1.5^{\mathrm{a}}$ & $-1 \cdot 3^{a}$ & 1.5 & 17.9 & $<0.001$ \\
\hline Regular-energy, $20 \mathrm{~g} / \mathrm{sip}$ & $-3 \cdot 2^{b}$ & 1.4 & -1.0 & $1.7^{\mathrm{a}}$ & $-1.4^{a}$ & 1.3 & 39.0 & $<0.001$ \\
\hline No-energy, $5 \mathrm{~g} / \mathrm{sip}$ & $-3 \cdot 1^{b}$ & 1.5 & $-1 \cdot 1$ & $1.6^{\mathrm{a}}$ & $-1.0^{\mathrm{a}}$ & 1.2 & $36 \cdot 7$ & $<0.001$ \\
\hline No-energy, $20 \mathrm{~g} / \mathrm{sip}$ & $-3 \cdot 0^{\mathrm{b}}$ & 1.5 & -0.9 & $1 \cdot 8^{\mathrm{a}}$ & $-1.5^{\mathrm{a}}$ & 1.7 & $27 \cdot 3$ & $<0.001$ \\
\hline \multicolumn{9}{|l|}{ Pleasantness } \\
\hline Regular-energy, $5 \mathrm{~g} / \mathrm{sip}$ & -0.9 & 1.2 & -0.7 & 1.3 & -0.7 & 1.3 & 0.4 & 0.68 \\
\hline Regular-energy, $20 \mathrm{~g} / \mathrm{sip}$ & $-1 \cdot 3^{b}$ & 1.3 & $-0 \cdot 8^{\mathrm{a}, \mathrm{b}}$ & 1.6 & $-0.7^{a}$ & $1 \cdot 1$ & 3.6 & 0.03 \\
\hline No-energy, $5 \mathrm{~g} / \mathrm{sip}$ & $-1 \cdot 3^{b}$ & 1.2 & $-0.7^{\mathrm{a}}$ & $1 \cdot 2$ & $-0.5^{a}$ & 1.4 & 5.8 & 0.004 \\
\hline No-energy, $20 \mathrm{~g} / \mathrm{sip}$ & $-1 \cdot 0^{b}$ & $1 . \overline{5}$ & $-0.4^{\mathrm{a}}$ & $1 \cdot 2$ & $-0.4^{a}$ & 1.6 & 5.5 & 0.05 \\
\hline
\end{tabular}

a,b Mean values within a row with unlike superscript letters were significantly different $(P<0.05)$.

${ }^{*}$ Ratings on a nine-point scale anchored from $1=$ not at all desire/pleasant $/$ much to $9=$ extremely desire/pleasant $/$ much.

$\dagger F$ value of the ANOVA comparing the decline in subjective change ratings of wanting and liking after orangeade consumption for each stimulus. All tests have 2, $104 \mathrm{df}$, except for the tests in the regular-energy, $20 \mathrm{~g} / \mathrm{sip}$ condition, which has $2,98 \mathrm{df}$.

orangeade. Despite the lower intake associated with small sips, subjective SSS was comparable between the two sip size conditions. Thus, the present results show that beverage consumption with small sips can lower intake, while the subjective sensory satiation remains high.

The effect of sip size may be explained by the duration of oro-sensory exposure (i.e. longer oro-sensory exposure for small sips). This may also explain the results of previous studies. Some of these demonstrated that chewing time was positively associated with $\mathrm{SSS}^{(23)}$ or negatively with ad libitum intake of a subsequent meal ${ }^{(24)}$. Others showed that viscous drinkable foods provide higher satiety ratings or a lower ad libitum intake than more liquid foods ${ }^{(7,25-27)}$. One study showed that soups, which were consumed with a spoon, elicited stronger feelings of satiety than beverages, which were drank normally ${ }^{(28)}$.

Apart from the duration of oro-sensory exposure, the differences in intake and/or perceived satiation between conditions in each of these previous studies could also be explained by other factors, such as a difference in chewing effort ${ }^{(29)}$, or differences in the cognitive impression that the stimuli impart to consumers (i.e. learned satiation: consumers expect thicker foods to be more satiating than more liquid foods, or soups to be more satiating than beverages $)^{(30,31)}$. A clear strength of the present study was that in none of the sip size conditions was chewing effort required for consumption, and that the orangeades imparted the same cognitive impression about energy content to the subjects in each of the sip size conditions. Thus, a difference in the duration of oro-sensory exposure is left as the only likely explanation for the difference in intake between sip size conditions. Yet, an independent effect of a difference in the number of exposures (i.e. the number of sips) per min on the difference in intake cannot be ruled out.

In the large sip condition, intake of the regular-energy orangeade was higher than intake of the no-energy orangeade, while subjective SSS was comparable between the two types of orangeade. In the small sip condition, on the other hand, subjective SSS based on the desire to drink was lower for the regular-energy than for the no-energy orangeade (in noenergy soft drink consumers only), while intake was comparable between the two orangeade types. These results both
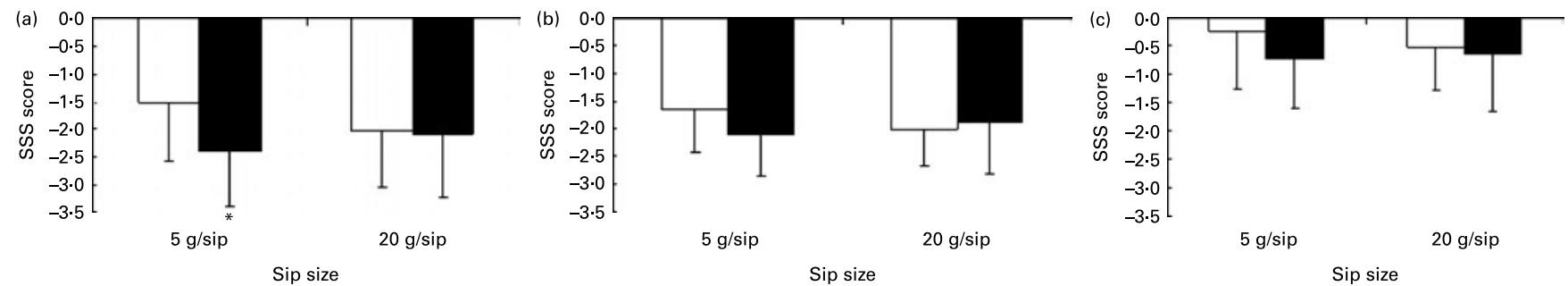

Fig. 3. Subjective sensory-specific satiation (SSS) for each of the three measures (desire (a), prospective consumption (b), liking (c)) in each sip size condition of the two types of orangeade ( $\square$, regular-energy; $\mathbf{\square}$, no-energy). For each of the measures perceived sensory satiation was calculated as the decline in the rating of the consumed orangeade - average decline in the rating of the reference stimuli (tomato juice and milk). Values are means, with standard deviations represented by vertical bars. Fisher's least significant difference (LSD) multiple comparisons were made among the four experimental conditions. * Mean value was significantly different from that of the regular-energy, $5 \mathrm{~g} / \mathrm{sip}$ condition $(P<0.05$; Fisher's LSD test). 
Table 3. Changes in appetite ratings from before to after ad libitum orangeade consumption in the four experimental conditions $\dagger$

(Mean values and standard deviations)

\begin{tabular}{|c|c|c|c|c|c|c|c|c|}
\hline & \multicolumn{2}{|c|}{$\begin{array}{c}\text { Regular- } \\
\text { energy, } 5 \mathrm{~g} / \mathrm{sip}\end{array}$} & \multicolumn{2}{|c|}{$\begin{array}{l}\text { Regular- } \\
\text { energy, } \\
20 \text { g/sip }\end{array}$} & \multicolumn{2}{|c|}{$\begin{array}{l}\text { No-energy, } \\
5 \mathrm{~g} / \mathrm{sip}\end{array}$} & \multicolumn{2}{|c|}{$\begin{array}{l}\text { No-energy, } \\
20 \text { g/sip }\end{array}$} \\
\hline & Mean & $\mathrm{SD}$ & Mean & SD & Mean & SD & Mean & SD \\
\hline Change in hunger & $-1 \cdot 2^{\star}$ & 1.4 & -1.6 & 1.5 & -1.6 & 1.6 & -1.4 & 1.3 \\
\hline Change in thirst & -3.4 & $1 \cdot 7$ & $-3 \cdot 2$ & $1 \cdot 7$ & $-3 \cdot 2$ & $1 \cdot 8$ & -3.4 & $2 \cdot 1$ \\
\hline Change in fullness & $2 \cdot 2$ & $1 \cdot 7$ & $2 \cdot 1$ & $1 \cdot 8$ & 1.8 & $1 \cdot 8$ & 1.5 & 1.9 \\
\hline Change in desire to eat & -1.4 & 1.7 & -1.4 & 1.4 & -1.5 & 1.6 & $-1 \cdot 2$ & 1.6 \\
\hline Change in desired amount to consume & $-1 \cdot 2^{\star}$ & $1 \cdot 3$ & -1.6 & 1.4 & $-1 \cdot 3$ & 1.5 & $-1 \cdot 0^{*}$ & $1 \cdot 8$ \\
\hline
\end{tabular}

* Mean value was significantly different from that of the regular-energy, $20 \mathrm{~g} / \mathrm{sip}$ condition $(P<0.05$; Fisher's least significant difference test). $\dagger$ Ratings on a nine-point scale anchored from $1=$ not at all to $9=$ extremely.

suggest that the development of SSS is attenuated for regularenergy orangeade compared with no-energy orangeade. This is not supported by most previous studies, which found that the degree of SSS of sweet stimuli is not dependent on the energy content $^{(18,19,32)}$. However, in support of the present results, a recent human neuroimaging study showed differential brain responses to energy-containing (sucrose) and no-energy sweeteners (sucralose). Only sucrose engaged activation of dopaminergic midbrain areas in correlation with subjective ratings of pleasantness ${ }^{(33)}$. A recent study showed that mice that lack the ability to taste sweetness developed a robust preference for energy-containing sweet stimuli over water, but not for no-energy sweet stimuli. This suggests that energy content can be detected even when no taste transduction is present $^{(34)}$. Thus, backed up by these findings ${ }^{(33,34)}$, the present results suggest that individuals are able to distinguish the sensory properties of energy-containing sweet foods from those of no-energy sweet foods and adjust their intake on the basis of that information.

The finding that subjective SSS (in the small sip condition) was lower for regular-energy orangeade than for no-energy orangeade among no-energy soft drink consumers only (not for regular-energy soft drink consumers) suggests that the ability to distinguish between sucrose and no-energy sweeteners requires repeated experience with no-energy sweet foods (i.e. learning of a combination of sweet taste and subsequent metabolic consequences). In support of these results, a human study ${ }^{(35)}$ showed that regular consumers of beverages sweetened with synthetic sweeteners lacked the increase in appetite in response to sweet taste, which was found in low consumers of beverages sweetened with synthetic sweeteners. On the other hand, previous rat studies suggested that a sensory distinction between energy-containing and no-energy sweet foods cannot be made, and that therefore eating sweet no-energy substances may degrade the predictive relationship between sweet taste and energy, leading to deficits in energy regulation ${ }^{(36-38)}$.

In the large sip condition perceived sweetness of the noenergy orangeade increased. This could therefore be an alternative explanation for the lower intake of no-energy orangeade compared with regular-energy orangeade. However, although significantly different from zero, the mean increase in perceived sweetness was only 0.4 on a nine-point scale. Therefore, it seems unlikely that the increased perceived intensity can explain the lower intake of no-energy orangeade.
In conclusion, we showed that small sip size, i.e. an increased duration of oro-sensory exposure, can diminish the intake of soft drinks. Moreover, the results suggest that with large sip size only the intake of energy-containing sweet beverages is higher than of no-energy sweet beverages. This underpins the importance of oral exposure time and SSS for meal termination. It may be that consumption of beverages with devices that reduce sip size, such as narrow straws or bottles with limited openings, may help to promote satiation, and thus to limit energy intake.

\section{Acknowledgements}

The present study was financially supported by Suikerstichting Nederland (The Netherlands Sugar Foundation). We gratefully acknowledge United Soft Drinks for producing and donating the syrups.

The main investigator of the present study was P. W., who wrote the study protocol and carried out the main body of the work, interpreted the results and wrote a large part of the manuscript. P. A. M. S. contributed to protocol writing, processing the results and writing the manuscript. Protocol writing, data collection and writing the manuscript were done under general supervision of C. de G.

The authors had no conflicts of interest.

\section{References}

1. Mela DJ (2006) Eating for pleasure or just wanting to eat? Reconsidering sensory hedonic responses as a driver of obesity. Appetite 47, 10-17.

2. Wansink B (2004) Environmental factors that increase the food intake and consumption volume of unknowing consumers. Annu Rev Nutr 24, 455-479.

3. Blundell JE, Green S \& Burley V (1994) Carbohydrates and human appetite. Am J Clin Nutr 59, 728S-734S.

4. Rolls BJ, Rolls ET, Rowe EA, et al. (1981) Sensory specific satiety in man. Physiol Behav 27, 137-142.

5. Sørensen LB, Møller P, Flint A, et al. (2003) Effect of sensory perception of foods on appetite and food intake: a review of studies on humans. Int J Obes 27, 1152-1166.

6. Weijzen PLG, Zandstra EH, Alfieri C, et al. (2008) Effects of complexity and intensity on sensory specific satiety and food acceptance after repeated consumption. Food Qual Prefer 19, 349-359. 
7. Zijlstra N, Mars M, De Wijk RA, et al. (2008) The effect of viscosity on ad libitum food intake. Int J Obes 32, 676-683.

8. Haber GB, Heaton KW, Murphy D, et al. (1977) Depletion and disruption of dietary fibre. Lancet ii, 679-682.

9. Hulshof T, De Graaf G \& Weststrate JA (1993) The effects of preloads varying in physical state and fat content on satiety and energy intake. Appetite 21, 273-286.

10. Mattes RD (1996) Dietary compensation by humans for supplemental energy provided as ethanol or carbohydrates in fluids. Physiol Behav 59, 179-187.

11. DiMeglio DP \& Mattes RD (2000) Liquid versus solid carbohydrate: effects on food intake and body weight. Int $J$ Obes Relat Metab Disord 24, 794-800.

12. Mattes RD (2006) Beverages and positive energy balance: the menace is the medium. Int $J$ Obes 30, S60-S65.

13. Spiegel TA \& Jordan HA (1978) Effects of simultaneous oral-intragastric ingestion on meal patterns and satiety in humans. J Comp Physiol Psychol 92, 133-141.

14. Spiegel T (2000) Rate of intake, bites, and chews - the interpretation of lean-obese differences. Neurosci Biobehav Rev 24, 229-237.

15. German RZ, Crompton AW, Owerkowicz T, et al. (2004) Volume and rate of milk delivery as determinants of swallowing in an infant model animal (Sus scrofia). Dysphagia 19, $147-154$.

16. De Graaf C, Schreurs A \& Blauw YH (1993) Short-term effects of different amounts of sweet and nonsweet carbohydrates on satiety and energy intake. Physiol Behav 54, 833-843.

17. Johnson J \& Vickers Z (1993) Effects of flavour and macronutrient composition of food servings on liking, hunger, and subsequent intake. Appetite 21, 25-39.

18. Rolls BJ, Hetherington M \& Burley VJ (1988) Sensory stimulation and energy density in the development of satiety. Physiol Behav 44, 727-733.

19. Rolls BJ, Laster LJ \& Summerfelt A (1989) Hunger and food intake following consumption of low-calorie foods. Appetite 13, 115-127.

20. Miller DL, Hammer VA, Peters JC, et al. (1996) Influence of the fat content of a food on sensory-specific satiety. FASEB $J$ 10, A 824 .

21. Miller DL, Bell EA, Pelkman CL, et al. (2000) Effects of dietary fat, nutrition labels, and repeated consumption on sensory-specific satiety. Physiol Behav 71, 153-158.

22. Van Strien T (2002) Dutch Eating Behaviour Questionaire Manual. Bury St Edmunds, UK: Thames Valley Test Company Ltd.
23. Hetherington MM \& Boyland E (2007) Short-term effects of sugar gum on snack intake and appetite. Appetite 48, 397-401.

24. Lavin JH, French SJ, Ruxton CHS, et al. (2002) An investigation of the role of oro-sensory stimulation in sugar satiety. Int $J$ Obes 26, 384-388.

25. Mattes RD \& Rothacker D (2001) Beverage viscosity is inversely related to postprandial hunger in humans. Physiol Behav 74, 551-557.

26. Tsuchiya A, Almiron-Roig E, Lluch A, et al. (2006) Higher satiety ratings following yoghurt consumption relative to fruit drink or dairy fruit drink. $J$ Am Diet Assoc 106, 550-557.

27. Harper A, James A, Flint A, et al. (2007) Increased satiety after intake of a chocolate milk drink compared with a carbonated beverage, but no difference in subsequent ad libitum lunch intake. Br J Nutr 97, 579-583.

28. Mattes RD (2005) Soup and satiety. Physiol Behav 83, 739-747.

29. Oka K, Sakuarae A, Fuijse T, et al. (2003) Food texture differences affect energy metabolism in rats. J Dent Res 82, 491-494.

30. Tournier A \& Louis-Sylvestre J (1991) Effect of the physical state of a food on subsequent intake in human subjects. Appetite 16, 17-24.

31. De Castro JM (1993) The effects of the spontaneous ingestion of particular foods or beverages on the meal pattern and overall nutrient intake of humans. Physiol Behav 53, 1133-1144.

32. Raben A, Vasilaras TH, Møller AC, et al. (2002) Sucrose compared with artificial sweeteners: different effects on ad libitum food intake and body weight after $10 \mathrm{wk}$ of supplementation in overweight subjects. Am J Clin Nutr 76, 721-729.

33. Frank GKW, Oberndorfer TA, Simmons AN, et al. (2008) Sucrose activates human taste pathways differently from artificial sweeteners. Neuroimage 39, 1559-1569.

34. De Araujo IE, Oliveira-Maia AJ, Sotnikova TD, et al. (2008) Food reward in the absence of taste receptor signaling. Neuron 57, 930-941.

35. Appleton KM \& Blundell JE (2007) Habitual high and low consumers of artificially-sweetened beverages: effects of sweet taste and energy on short-term appetite. Physiol Behav 92, 479-486.

36. Davidson TL \& Swithers SE (2004) A Pavlovian approach to the problem of obesity. Int J Obes 28, 933-935.

37. Swithers SE \& Davidson TL (2005) Obesity: outwitting the wisdom of the body? Curr Neurol Neurosci Rep 5, 159-162.

38. Swithers SE \& Davidson TL (2008) A role for sweet taste: calorie predictive relations in energy regulation by rats. Behav Neurosci 122, 161-173. 